\title{
Visualisation and measurement of a laminar boundary layer on the surface of an isothermal section-triangular roof
}

Haoyu Zhai ( 17115269@bjtu.edu.cn )

Beijing Jiaotong University

Juan F. Torres

Australian National University https://orcid.org/0000-0002-3054-8638

Yongling Zhao

ETH Zürich https://orcid.org/0000-0003-3492-0844

\section{Feng Xu}

Beijing Jiaotong University https://orcid.org/0000-0002-0720-4247

\section{Research Article}

Keywords: Natural convection, uncontrolled disturbance, phase-shifting interferometry, flow visualisation

Posted Date: July 23rd, 2021

DOI: https://doi.org/10.21203/rs.3.rs-731106/v1

License: (9) This work is licensed under a Creative Commons Attribution 4.0 International License. Read Full License 


\section{Abstract}

Natural convection in air over a heated section-triangular roof with a fixed aspect ratio of 0.1 is experimentally investigated. The development of the flow over the roof subject to a range of temperatures is measured by digital interferometry and thermocouples. The experiments present distinct images of the thermal boundary layer, which changes from a quasi-steady to an unsteady state as the surface temperature of the triangular roof increases. Contrary to numerical simulations previously published, the observed flow becomes unsteady, which is very likely influenced by uncontrolled perturbations at the critical Rayleigh number where a pitchfork bifurcation of a steady flow is theoretically expected.

\section{Introduction}

Natural convection from shortwave radiation heated rooftops plays a significant role in thermal management of built environment and has been widely investigated in building ventilation [2], solar energy $[4,6,7]$ and indoor energy conservation $[1,3,5]$. However, less attention has been paid to fundamental research in flow dynamics and heat transfer. Our previous theoretical studies predict the mechanism of dynamics and heat transfer for the flow over the roof in laminar regimes under different heating condition $[19,17]$ and subsequent flow bifurcations with increasing of Rayleigh number (Ra) [18]. However, the static wind condition used in these theoretical studies is not achievable in buildings surrounded by an atmospheric boundary layer. This motivates us to conduct laboratory scale experiments on roof to further investigate its dynamics and heat and mass transfer where perturbation exists.

This experiment investigates the flow structure over an inclined roof. For non-isothermal visualisation experiments with open boundaries in air, it is challenging to use particle image velocimetry (PIV). Instead, phase-shifting interferometry (PSI) can be used to visualise temperature fields. External thermocouples can also assist to monitor the temperature time series. As a visualisation method, PSI has been successfully used in heat and mass transfer experiments with both closed and open boundaries $[9,10$, $11,14,13,15]$. In this study, a description to thermal boundary layer over the roof is presented together with temperature signals that indicate the symmetric flow structure in steady state. Fluctuation of thermal boundary layer induced by an uncontrolled and external perturbation is also taken into consideration.

This experimental work focuses on the flow structure in steady state, from conduction-dominant to convection-dominant regime. The aim is to develop a reliable experimental method to investigate flow transition atop a heated section-triangular roof with fixed aspect ratio.

\section{Experiment Setup}

\section{Experiment model}

The schematic of a copper block designed as a roof model is presented in figure 1(a), with $60 \mathrm{~mm}$ length, $60 \mathrm{~mm}$ width and $23 \mathrm{~mm}$ height. In this block, $3 \mathrm{~mm}$ measuring from the top is the model as a roof with 
aspect ratio 0.1 where the characteristic length is half width of the roof, $20 \mathrm{~mm}$ remaining is applied to heat the copper block uniformly. As shown in figure 1(b), a stand made of thermoplastic polyester (polylactide; PLA) is used to support the copper block. A proportional-integral-derivative (PID) control system comprised of a Peltier module, fins, and a cooling fan (turned off during a measurement), is placed underneath the copper block to heat and regulate its temperature during the experiment. To reduce the uncontrolled perturbation from the ventilation system in the experimental room, the cover shown in figure 1(c) is placed above the copper block and its lateral walls have two empty holes to allow the passage of the test beam (see details of PSI in [15], a similar cover was also used in [8]). For this reason, an uncontrolled disturbing flow will still come into the cover along the horizontal direction. Multiple thermocouples are also fixed above the copper block to monitor the temperature time series at geometrically symmetric locations.

\section{Digital interferometry}

A previously developed temporal phase-shifting interferometry technique was implemented here to investigate natural convection (see [15] for details). The laser beam first passes through a neutral-density (ND) filter to adjust the beam intensity while a polarising beam splitter (PBS) divides the attenuated laser beam into a test beam and a reference beam. The beam passes through a refractive-index field caused by the non-isothermal flow rising from the roof model. Then, the 'undisturbed' reference beam wavefront and deformed test beam wavefront are merged by a second PBS. The beams polarisation is changed from linear to circular polarisations by using a quarter-wave plate, and finally the light is filtered by a linear polariser at controlled angles before the interferogram is recorded by a CMOS camera. These interferograms represent the average temperature along the optical path. To obtain a temperature field with high spatial resolution, a three-bucket phase-shifting equation is used to obtain the phase-shifted data, which is unwrapped by an image processing program. Note that an isothermal background image (before heating) is needed [15].

To acquire the temperature field from wrapped phase-shifting images, a two boundary condition method is used [10]. First, we select the layers of the phase-shifted data in both non-isothermal (natural convection) and background phase-shifted data shown in figures 2(a) and (b), respectively. Next, these phase-shifted data are unwrapped to produce the phase maps shown in figures 2 (c) and (d). Then, the isothermal unwrapped data will be subtracted with background unwrapped data to obtain the phase map in figure 2(e), which is produced by the temperature field (without major optical aberrations). Finally, we use the two boundary condition method to determine the brightest pixel as maximum temperature (heating temperature) and the darkest pixel as minimum temperature (ambient temperature) in experiment, and the actual temperature field is plotted in figure 2(f).

\section{Experiment conditions}

In the experiments, the temperature difference between the copper block and ambient air ranged from $5 \mathrm{~K}$ to $30 \mathrm{~K}$. The flow structures on $Y-Z$ and $X-Y$ planes (see coordinates in figure $1(\mathrm{a})$ ) are presented 
together with the temperature time series on three points along a $Z$ direction. The primary nondimensional physical parameters is the Rayleigh number Ra [10], defined in equation (1) as:

$$
\mathrm{Ra}=\frac{g \beta \Delta T l^{3}}{v \kappa},
$$

the characteristic length / is half width of copper block and temperature difference $\Delta T$ is the difference between heating copper block and ambient air. When the experiment results are compared with numerical simulation results in Table 1, nondimensional temperature is used, defined in equation (2):

$$
\Theta=\frac{T_{\mathrm{m}}-T_{0}}{\Delta T}
$$

where $T_{\mathrm{m}}$ means the temperature monitored by thermocouples and $T_{0}$ means the temperature of ambient air.

\section{Results}

\section{Thermal boundary layer}

For the results shown in figure 3 , the field of view is located at the right centre on the $Y-Z$ plane, indicated in figure 3(d). The flow is under a conduction-dominant regime (negligible flow) at $\Delta T=5 \mathrm{~K}$ for Ra $=1.3 \times$ $10^{4}$ in figure 3(a), as a marginal inclination results in a weak flow even when $\mathrm{Ra} \rightarrow 0[16,12,18]$.

Furthermore, with the increasing of temperature difference to $\Delta T=7.5 \mathrm{~K}\left(\mathrm{Ra}=1.99 \times 10^{4}\right)$, as shown in in figure $3(\mathrm{~b})$, the convection gradually dominates the flow dynamics as a measurable thermal boundary layer forms over the surface. Further increasing the temperature to $\Delta T=10 \mathrm{~K}\left(\mathrm{Ra}=2.6 \times 10^{4}\right)$, a distinct thermal boundary layer forms, as shown in figure 3(c) with $0.1 \mathrm{~K}$ temperature gradient per yellow line. Comparing figures $3(\mathrm{~b})$ and (c), we find that the thermal boundary layer appears to be paralleled with the surface at first and then slightly tilted from left to right. This indicates that the buoyancy force controls the flow structure inducing a plume downstream at the top right corner of the field of view. From the temperature field in figure 3(c), we noticed that the thickness of some yellow lines is thicker than others, which might have occurred due to transient perturbations. Temporally captured PSI image in the laboratory and detailed analysis are presented in the following subsection.

Corresponding numerical simulation results are used to validate the experimental observations. Figure 4(a) shows the temperature on selected $X$ locations of $Y-Z$ plane over the roof, which are then averaged to obtain the contour shown in figure 4(b). Figure 4(c) presents the numerical results showing the average temperature profile corresponding to the field of view in the experiment. Likewise, the temperature field was analysed along the $Z$ direction ( $X-Y$ plane) and plotted in figures $4(e)$ and (f) for the simulation and experimental results, respectively. This comparison shows that that the inner region of the thermal boundary layer under buoyancy-driven force fits qualitatively with the numerical results. A distinct plume 
stem was observed using PSI. However, we found a random, non-periodic break-form-break cycle phenomenon during the experiments, which is very likely caused by a surrounding ambient flow in the laboratory space that cause the top of the plume to be greatly perturbed.

\section{Temperature series}

Thermocouples are placed within the thermal boundary layer to measure the temperature time series, and the measurement positions are indicated in figures $5(a)$ and (b). The results of the case with the temperature difference of $10 \mathrm{~K}$ are shown as an example in figure 5 (c) at the position B1 and B3. The temperature time series obtained from both measurements are almost identical at the beginning $(0 \mathrm{~s}-20$ s) of the experiment, and the flow is in a quasi-steady state. When an external perturbation occurs between $30 \mathrm{~s}$ and $130 \mathrm{~s}$, the two temperature time series suggest that the thermal boundary layer fluctuates with their spanwise temperature peaks appearing almost simultaneously. Our observations indicate that the thick yellow lines in figure 3(c) occur during this thermal boundary layer fluctuation. After the ambient flow passing through, the thermal boundary layer become quasi-steady again. Table 1 reveals that the temperatures at these two points remains nearly the same with the increasing of $\mathrm{Ra}$, indicating that the flow structure is symmetric and in a quasi-steady state.

Table 1. Nondimensional mean temperature values at B1 and B3 for different Ra.

\begin{tabular}{|l|l|l|l|l|}
\hline Ra & $2.6 \times 10^{4}$ & $3.9 \times 10^{4}$ & $5.2 \times 10^{4}$ & $7.8 \times 10^{4}$ \\
\hline$\Theta_{\mathrm{B} 1}$ & 0.6524 & 0.5505 & 0.5478 & 0.5125 \\
\hline$\Theta_{\mathrm{B} 3}$ & 0.6423 & 0.5294 & 0.5227 & 0.5488 \\
\hline Deviation & $1.54 \%$ & $4 \%$ & $4.58 \%$ & $6.62 \%$ \\
\hline
\end{tabular}

This uncontrolled ambient flow may cause the asymmetric structure along the $x$ direction which is different with the results observed in figure 4(f). As shown in figure 6(a), the nondimensional mean temperature values at slice 2 (indicated in figure $5 \mathrm{~b}$ ) for the case at a temperature difference of $10 \mathrm{~K}$ indicate that the thermal boundary layer tilts to the left when the uncontrolled perturbation occurs. Tilted isotherms are observed in the PSI measurement shown in figure 6(b), which are consistent with the thermocouple measurement result. This asymmetry can promote an early transition $[12,16]$ when the temperature difference reaches $20 \mathrm{~K}$, which was confirmed with the thermocouple measurements, e.g. B2 shown in figure 6(c).

\section{Conclusions}

Natural convection on a section-triangular roof was experimentally investigated in a small scale (low $\mathrm{Re}$ ) laboratory environment. A distinct development of the thermal boundary layer from conduction dominance to convection dominance is observed as the temperature difference increase from $7.5 \mathrm{~K}$ to 10 $\mathrm{K}$, corresponding to $\mathrm{Ra}=1.99 \times 10^{4}$ and $2.6 \times 10^{4}$, respectively. The uncontrolled perturbations cause an 
asymmetric flow structure and a quasi-steady flow, whereas a symmetric flow structure and a steady flow was previously reported by numerical simulations. Nevertheless, experimental and simulation results have a qualitative agreement at the measured locations when perturbations are reduced. When the perturbations occur, the results obtained through the PSI visualisation and the thermocouple measurements are consistent.

\section{Declarations}

\section{Acknowledgements}

This work is supported financially by the by the National Natural Science Foundation of China (Grant No. 11972072). Funding from the 2019 ANU Global Research Partnership Scheme for the project "Exchange on multiscale convective heat transfer" is also acknowledged. Professor Atsuki Komiya from Tohoku University contributed to setting up the phase-shifting interferometer used in this research.

\section{Competing Interests}

The authors declare no competing interests.

\section{References}

1. Cao, X., Liu, J., Jiang, N. and Chen, Q., Particle Image Velocimetry Measurement of Indoor Airflow Field: A Review of the Technologies and Applications, Energy Build., 69, 2014, 367-380.

2. Fan, Y., Li, Y., Hang, J., Wang, K. and Yang, X., Natural Convection Flows along a 16-Storey High-Rise Building, Build. Environ., 107, 2016, 215-225.

3. Kumar, R. and Kaushik, S. C., Performance Evaluation of Green Roof and Shading for Thermal Protection of Buildings, Build. Environ., 40, 2005, 1505-1511.

4. Lei, Y., Zhang, Y., Wang, F. and Wang, X., Enhancement of Natural Ventilation of a Novel Roof Solar Chimney with Perforated Absorber Plate for Building Energy Conservation, Appl. Therm. Eng., 107, 2016, 653-661.

5. Owrak, M., Aminy, M., Jamal-Abad, M. T. and Dehghan, M., Experiments and Simulations on the Thermal Performance of a Sunspace Attached to a Room Including Heat-Storing Porous Bed and Water Tanks, Build. Environ., 92, 2015, 142-151.

6. Peng, C. and Yang, J., The Effect of Photovoltaic Panels on the Rooftop Temperature in the EnergyPlus Simulation Environment, Int. J. Photoenergy, 2016, 2016.

7. Phiraphat, S., Prommas, R. and Puangsombut, W., Experimental Study of Natural Convection in PV Roof Solar Collector, Int. Commun. Heat Mass Transf., 89, 2017, 31-38.

8. Saxena, A., Kishor, V., Srivastava, A. and Singh, S., Whole Field Measurements to Identify the Critical Rayleigh Number for the Onset of Natural Convection in Top Open Cavity, Exp. Heat Transf., 33, 2020, 
$123-140$.

9. Shoji, E., Komiya, A., Okajima, J., Kawamura, H. and Maruyama, S., High-Speed Phase-Shifting Interferometry Using Triangular Prism for Time-Resolved Temperature Measurement, Appl. Opt., 54, $2015,6297$.

10. Shoji, E., Komiya, A., Okajima, J. and Maruyama, S., Development of Quasi Common Path PhaseShifting Interferometer for Measurement of Natural Convection Fields, Int. J. Heat Mass Transf., 55, 2012, 7460-7470.

11. Shoji, E., Nakaoku, R., Komiya, A., Okajima, J. and Maruyama, S., Quantitative Visualization of Boundary Layers by Developing Quasi-Common-Path Phase-Shifting Interferometer, Exp. Therm. Fluid Sci., 60, 2015, 231-240.

12. Torres, J. F., Henry, D., Komiya, A. and Maruyama, S., Transition from Multiplicity to Singularity of Steady Natural Convection in a Tilted Cubical Enclosure, Phys. Rev. E, 92, 2015, 23031.

13. Torres, J. F., Komiya, A., Henry, D. and Maruyama, S., Measurement of Soret and Fickian Diffusion Coefficients by Orthogonal Phase-Shifting Interferometry and Its Application to Protein Aqueous Solutions, J. Chem. Phys., 139, 2013.

14. Torres, J. F., Komiya, A., Shoji, E., Okajima, J. and Maruyama, S., Development of Phase-Shifting Interferometry for Measurement of Isothermal Diffusion Coefficients in Binary Solutions, Opt. Lasers Eng., 50, 2012, 1287-1296.

15. Torres, J. F., Zhao, Y., Xu, S., Li, Z. and Komiya, A., Optical Method for Simultaneous High-Resolution Measurement of Heat and Fluid Flow: The Case of Rayleigh-Bénard Convection, Phys. Rev. Appl., 14, 2020, 54038.

16. Torres, J., Henry, D., Komiya, A. and Maruyama, S., Bifurcation Analysis of Steady Natural Convection in a Tilted Cubical Cavity with Adiabatic Sidewalls, J. Fluid Mech., 756, 2014, 650-688.

17. Zhai, H., Nie, B., Chen, B. and Xu, F., Unsteady Flows on a Roof Imposed by a Periodic Heat Flux: 2D Simulation and Scaling Analysis, Int. J. Therm. Sci., 145, 2019, 106002.

18. Zhai, H., Torres, J. F., Zhao, Y. and Xu, F., Transition from Steady to Chaotic Flow of Natural Convection on a Section-Triangular Roof, Phys. Rev. Fluids, 6, 2021, 1-24.

19. Zhai, H., Xu, F., Saha, S. C. and Hou, Y., Natural Convection and Heat Transfer on a Section-Triangular Roof, Int. Commun. Heat Mass Transf., 92, 2018, 23-30.

\section{Figures}


$(a)$

(b)

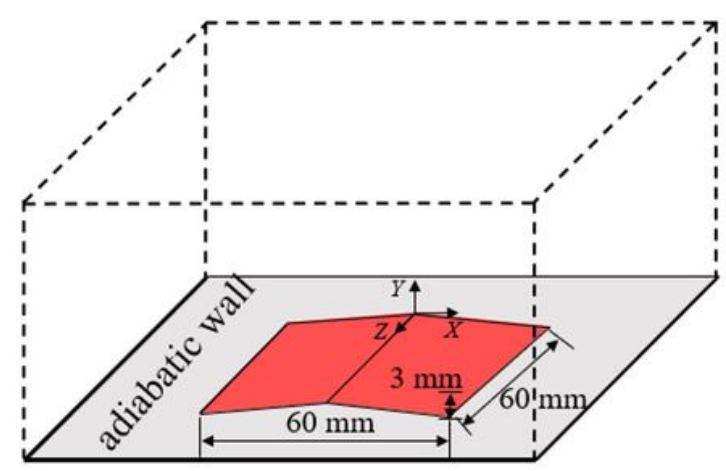

$(c)$

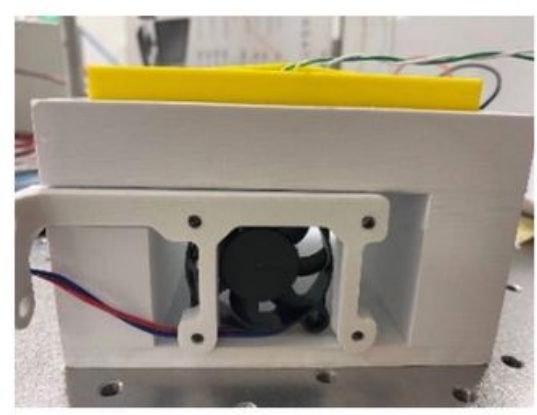

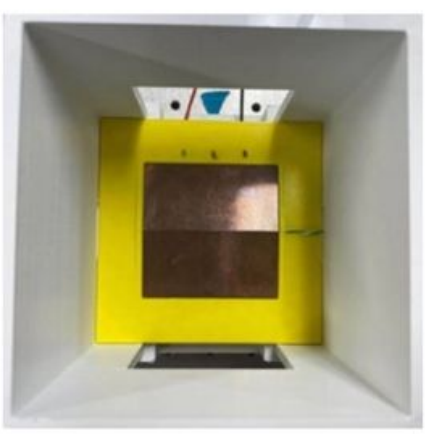

\section{Figure 1}

Experimental setup. (a) Schematic of experiment model. (b) Photograph showing the copper block and some parts of the temperature control system. (c) Top view photograph showing the cover and symmetrically placed thermocouples.

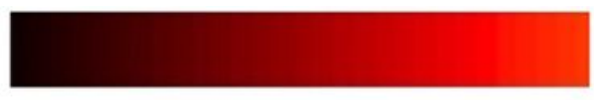

\section{Ambient temperature Heating temperature}

(a)

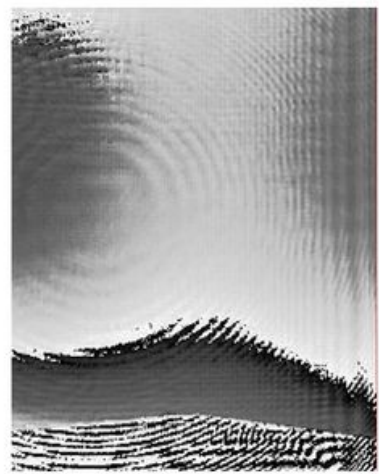

(b)

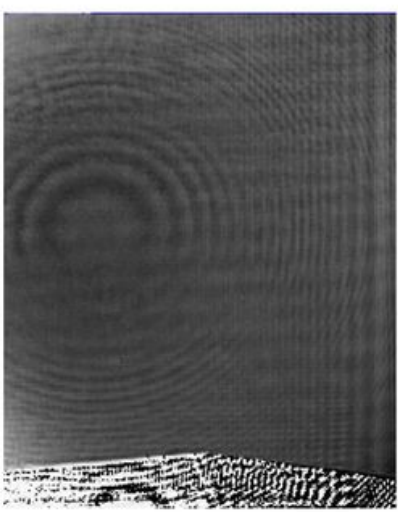

(c)

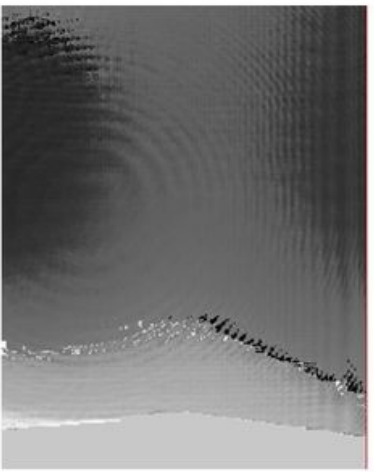

(d)

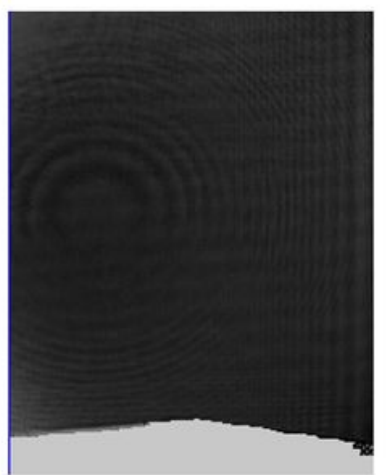

(e)

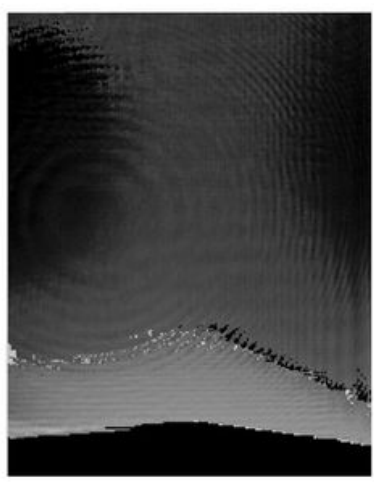

$(f)$

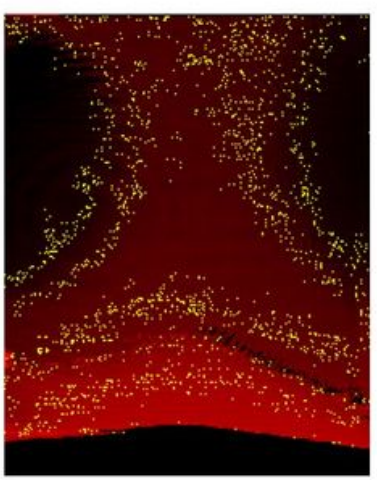

Figure 2 
Data processing for the case of temperature difference of $20 \mathrm{~K}$. (a), (b) Wrapped phase-shifting images for non-isothermal and isothermal background, respectively. (c), (d) Corresponding unwrapped phase map for (a) and (b). (e) Phase map after subtracting (d) from (c). (f) Actual temperature field.

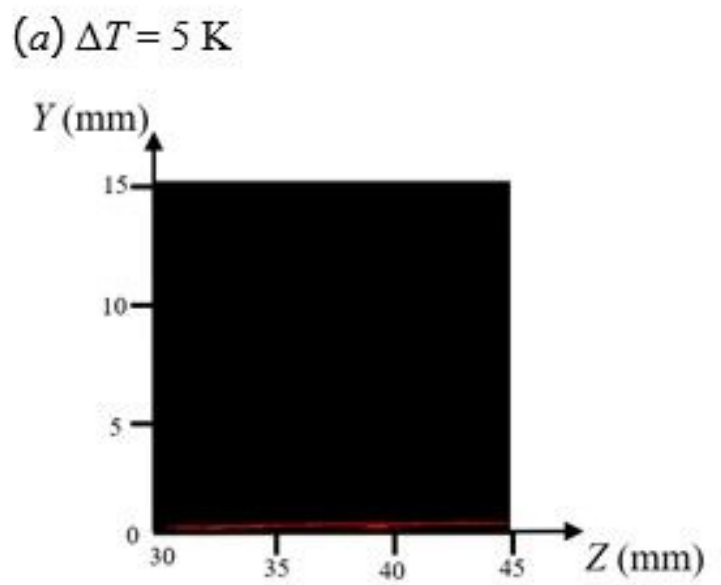

(c) $\Delta T=10 \mathrm{~K}$

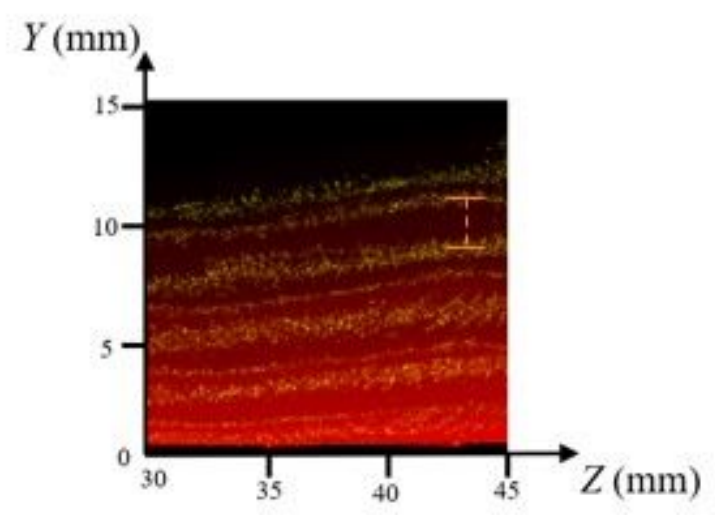

(b) $\Delta T=7.5 \mathrm{~K}$

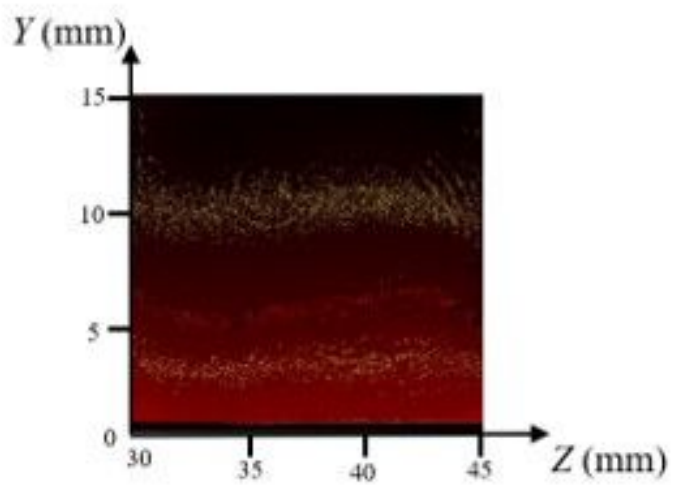

$(d)$

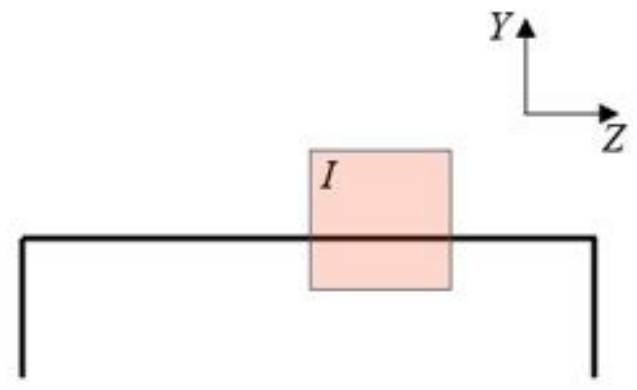

\section{Figure 3}

Visualisation experiment as the temperature difference is increased. Panels (a), (b) and (c) show the temperature fields for temperature difference at 5, 7.5 and $10 \mathrm{~K}$, respectively. (d) Schematic for the location of field of view or test beam. 
(a)

(b)
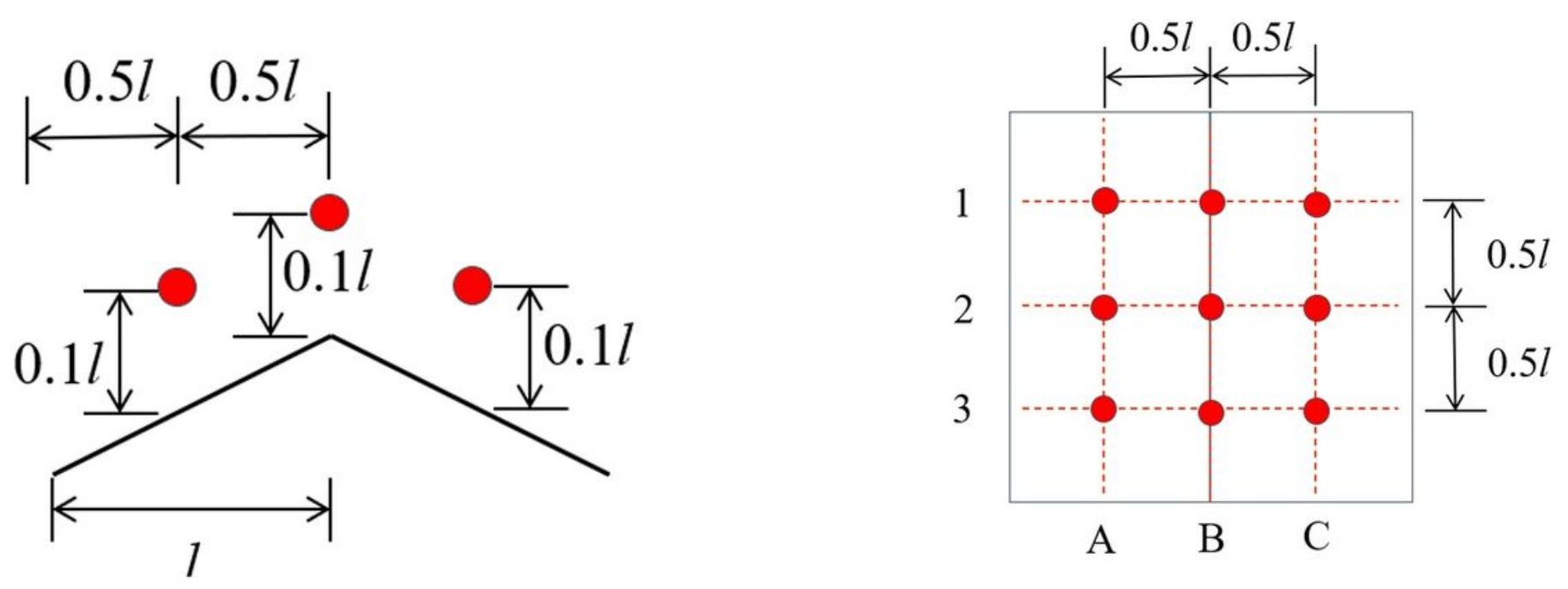

(c)

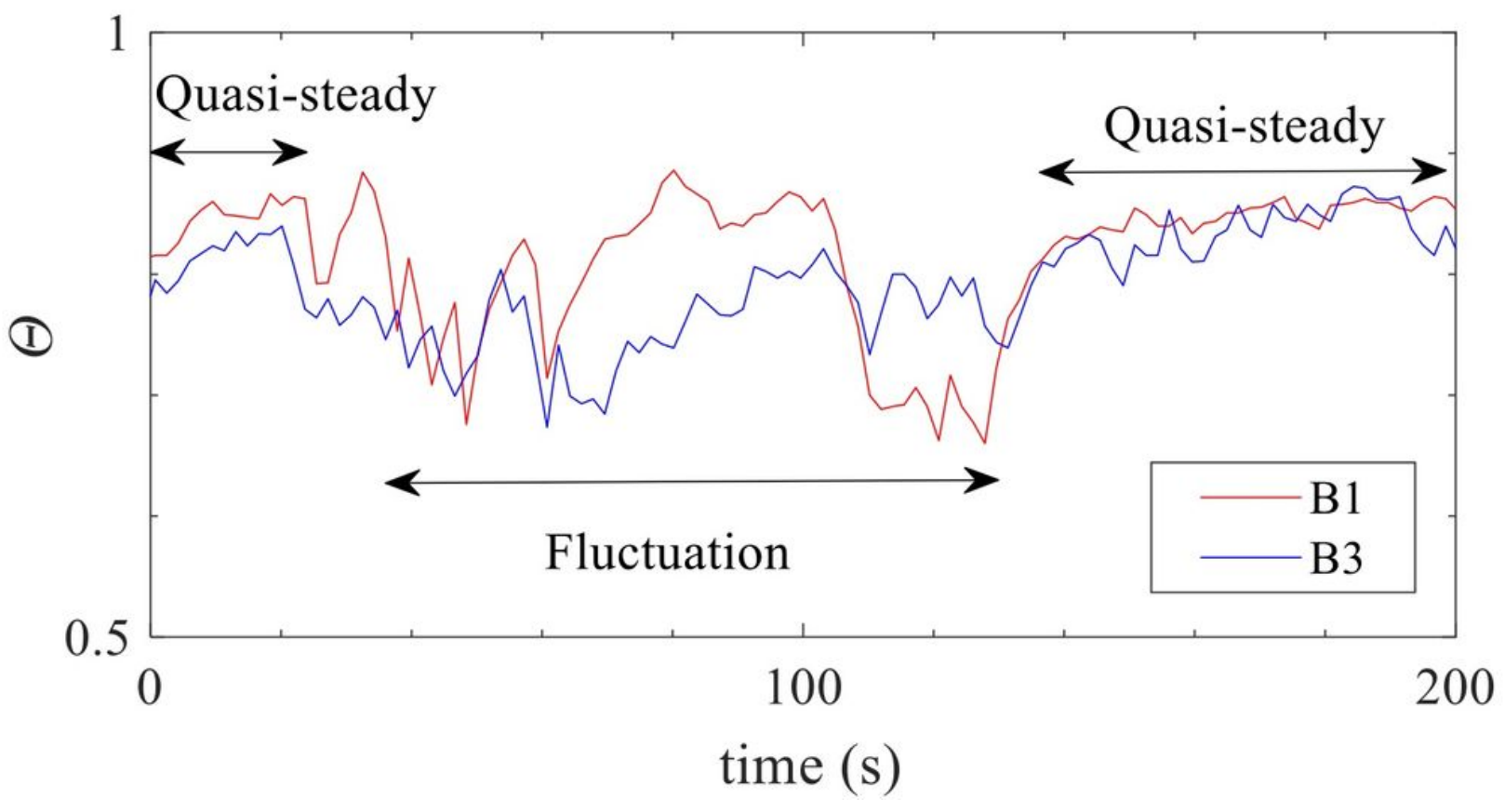

Figure 5

Measurement and visualisation results. (a) Nondimensional mean temperature values at slice 2 for temperature difference of $10 \mathrm{~K}$. (b) Experimental temperature fields for temperature difference at $20 \mathrm{~K}$ under uncontrolled perturbation effect in $\mathrm{X}-\mathrm{Y}$ plane. (c) Nondimensional temperature time series for a temperature difference of $20 \mathrm{~K}$ at B2. 

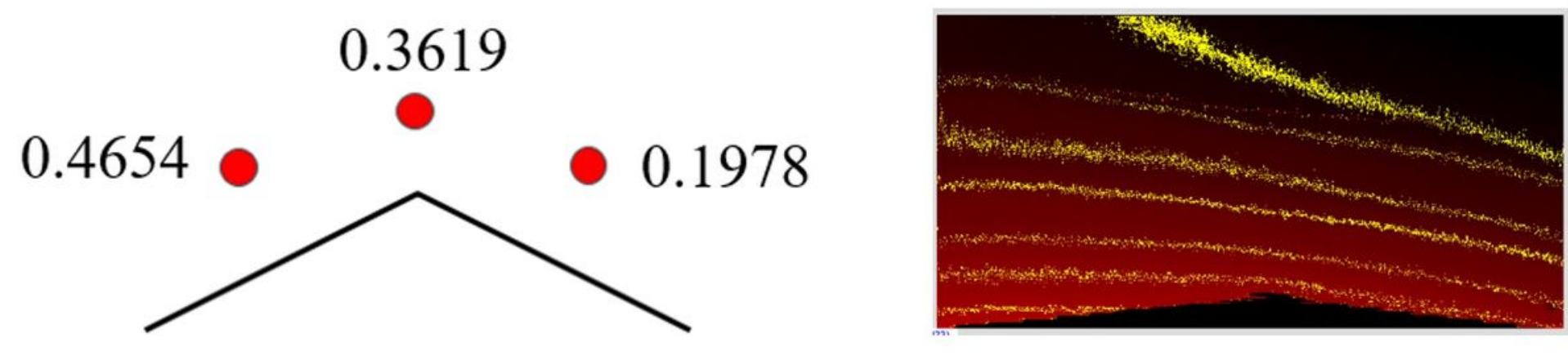

$(c)$

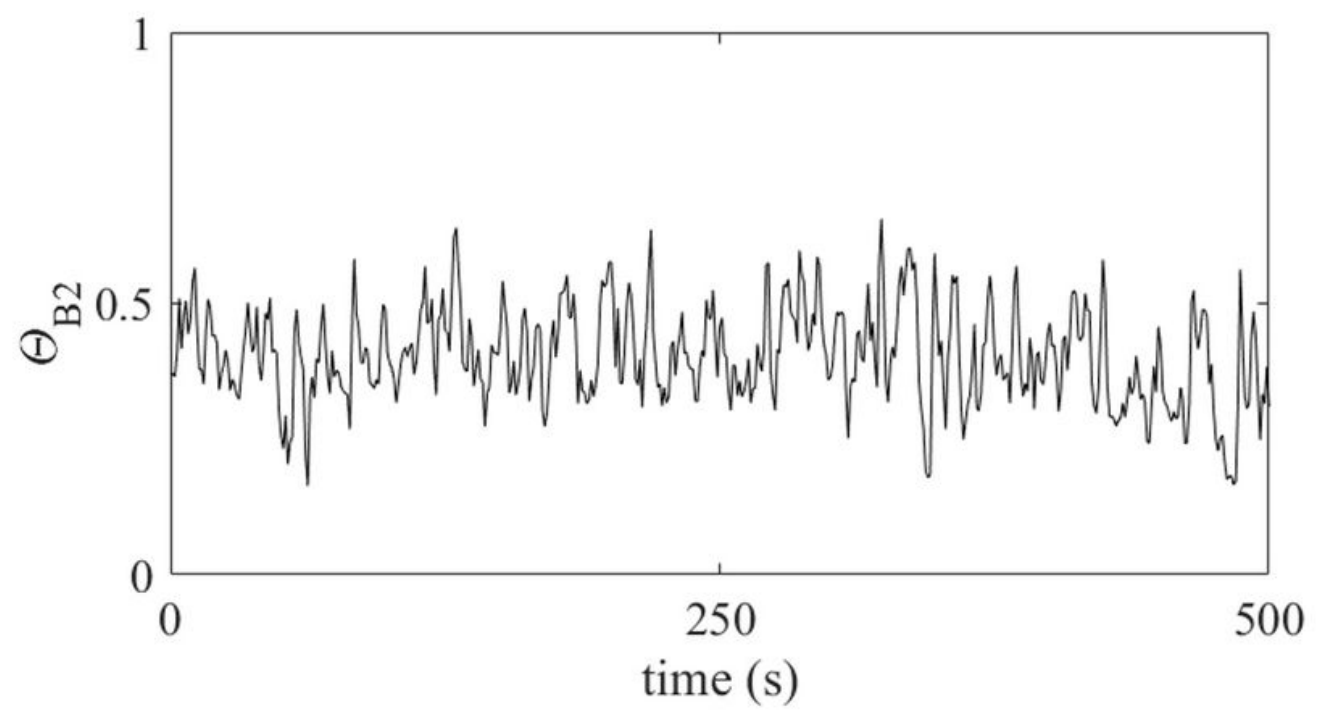

Figure 6

Temperature time series at geometrically symmetric locations. $(a, b)$ Schematic showing the position of thermocouples that monitored the temperature. (c) Nondimensional temperature time series for a temperature difference of $10 \mathrm{~K}$ at B1 and B3. 